\title{
Psychological and social evaluation in cases of deliberate self-poisoning seen in an accident department
}

\author{
R GARDNER, R HANKA, S J ROBERTS, J M ALLON-SMITH, A A KINGS, R NICHOLSON
}

\begin{abstract}
The outcome in 115 consecutive patients with mild self-poisoning seen by junior medical staff and discharged from the accident department was compared with that of 98 similar patients admitted to the medical wards. Psychiatrists saw only four patients in the accident department and 25 admissions. In making their assessments the junior medical staff considered psychosocial factors as well as the patients' physical condition. Most patients recommended for further care, and discharged from the accident department, subsequently received it. Repetition rates were similar in the two groups and there had been no suicides when patients were followed up at one year. It is feasible for junior staff in an accident department to decide whether patients with selfpoisoning need admission or may be discharged with or without subsequent referral for psychiatric or social work help.
\end{abstract}

\section{Introduction}

The Department of Health's recommendation ${ }^{12}$ that every self-poisoned patient should be assessed by a psychiatrist has been questioned in the case of patients admitted to medical wards. $^{34}$ At Addenbrooke's Hospital we have shown ${ }^{5-7}$ that assessment may be carried out by other doctors, and this has been accepted by the colleges of physicians and psychiatrists. ${ }^{8}$ The position is less clear for self-poisoned patients in accident departments, where official policy is that assessment may be carried out only by psychiatrists. Kreitman, ${ }^{9}$ however, questions whether it should be attempted at all, and advocates admission of all such patients. Few hospitals have enough acute medical beds for this purpose.

We have carried out a prospective study to see if it is feasible for junior medical staff in an accident department to decide whether patients with self-poisoning need admission to hospital or may be discharged with or without recommendations for psychiatric or social work referral.

\section{Patients and methods}

All patients with self-poisoning seen in the accident department of Addenbrooke's Hospital between 1 November 1978 and 31 May 1979 were included if they were over 14 years old and if they were conscious, or drowsy but conscious, on arrival. Assessment was carried out by senior house officers and nursing staff without special instruction, and by medical registrars. We decided against carrying out a randomised trial because of the shortage of acute medical beds. As patients were matched for levels of consciousness and were either admitted or discharged we compared these two groups. As we expected that admissions would far outnumber the patients with self-poisoning who were discharged, we selected a sample of the former by means of a table of random numbers. The consecutive series of patients who were discharged from the accident department are referred to as group D and the random sample of admissions to the medical wards as group A.

Patients in group D were interviewed by a research assistant, in their homes whenever possible, about three weeks after their discharge from the accident department, and a questionnaire was completed. Patients in group A were interviewed on the ward shortly after their admission using a similar questionnaire. Both groups gave permission for the research assistant to follow them up and for general practitioners and any other agencies to be contacted.

The study was terminated if any individual patient repeated the poisoning or self-injury or committed suicide, or if not, at the end of one year. The research assistant wrote to all patients and visited them, whenever possible, at home. She carried out structured interviews while general practitioners completed a short questionnaire at six months and one year. Hospital case notes were examined. When appropriate, social workers and probation officers were contacted and coroner's records scrutinised. Inquiries were made at the Office of Population Censuses and Surveys about all untraced patients. Data were analysed using $\chi^{2}$ test or Fisher's exact test where appropriate. The repetition rates were calculated using standard statistical techniques for follow-up studies-that is, the figures were corrected for patients who had been lost to follow-up for any reason.

\section{Results}

Altogether 314 patients were consecutively seen in the accident department (figure), of whom 115 were discharged and the remainder admitted. Ninety-eight of the 199 admissions were randomly selected for group A.

\section{ASSESSMENT OF PHYSICAL RISK}

There was no difference between the two groups with respect to age, sex, mode of referral, time and day of attending hospital, whether the act was one of self-poisoning or self-injury, the use of alcohol, or the particular drug(s) taken.

A higher proportion of admissions (group A) had taken three or more drugs and had had a stomach wash-out; more patients in group $\mathrm{D}$ had taken a single poison only. Both differences were statistically significant $(p<0.05)$. Admission was prompted by possible physical risk in two of the eight patients currently receiving psychiatric treatment in Fulbourn Hospital and in six of seven who 
had swallowed a non-ingestant (skin lotion, household bleach, ammonia, and weedkiller). As a result, group $\mathrm{A}$ included some patients selected for admission because of their physical condition, but this factor was unlikely to have accounted for almost twice as many admissions as discharges.

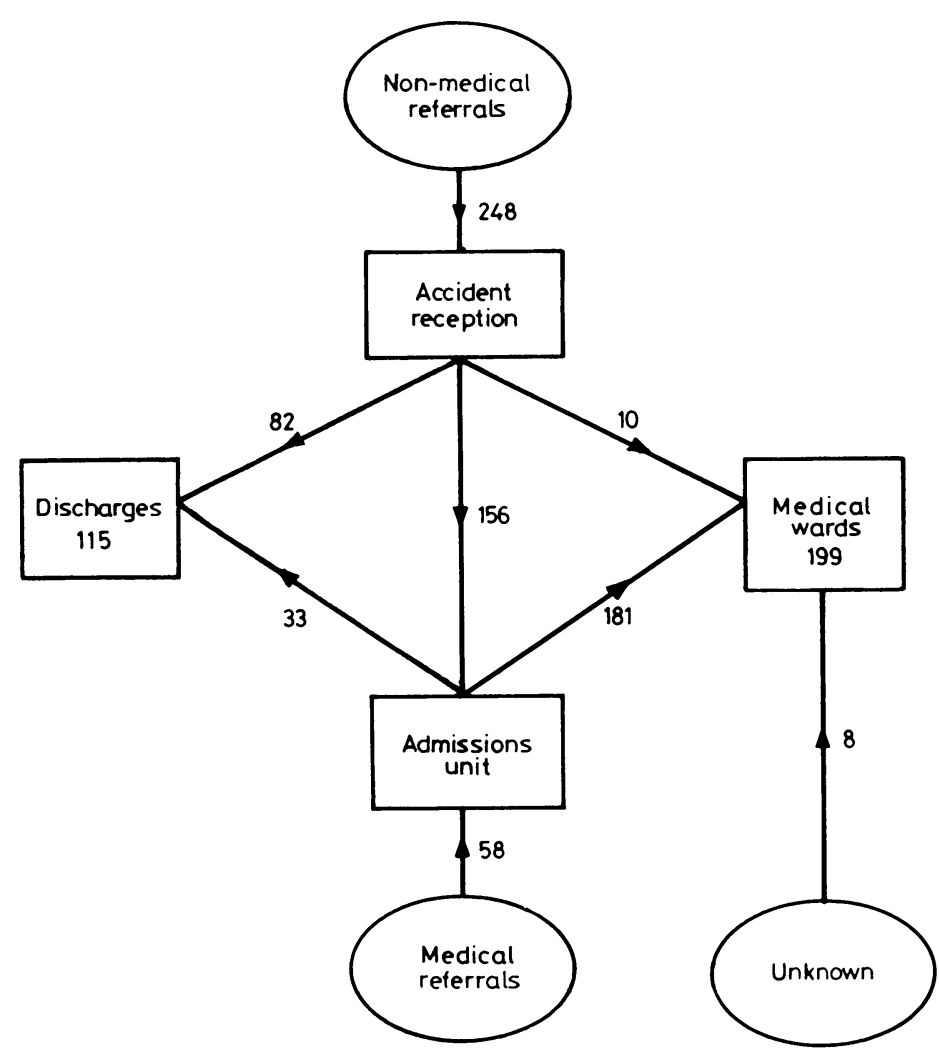

Fate of 314 consecutive cases of mild self-poisoning referred to Addenbrooke's Hospital, November 1978-May 1979.

\section{PSYCHOLOGICAL AND SOCIAL EVALUATION}

The two groups were similar with respect to social class, number and age of children, employment, criminal record, whether the overdose was the "first ever" or one of a number, whether patients had previously been admitted after self-poisoning, and how long ago this had occurred. The number of patients who had at some time received psychiatric treatment was comparable, but group D contained more patients currently receiving psychiatric treatment $(p<0.05)$. Nine suicidal patients, other than those currently receiving treatment in a psychiatric ward, were identified and were admitted informally to the psychiatric hospital.

Widowed and common-law marriages were more frequent in this group, while marital separation was more common in group A ( $p<0.05$ for each), though the reasons for the differences are obscure. A higher proportion of patients in group A were living at home, whereas in group D a higher proportion were living alone or in lodgings $(p<0.05)$. Twenty of these were discharged: seven declined admission and two of them refused a stomach wash-out; eleven were accompanied by a responsible adult. Most attended in daylight hours, and three of the four patients who came at night were observed for up to four hours.

On the wards consultant physicians asked psychiatrists (consultants or senior registrars) to see $25(25.5 \%)$ patients, in contrast to staff in the accident department who obtained a psychiatric opinion in only four $(3.5 \%)$ instances. We have no record of the number of times advice was obtained by telephone from the senior psychiatrist on call. The senior house officers referred $156(62.9 \%)$ patients to medical registrars in another part of the accident department (admissions unit) who, in turn, discharged 20 (figure).

Medical staff did offer patients in group D some psychiatric treatment and social work support (table I), but significantly more patients took their own discharge or were offered no treatment $(\mathrm{p}<0.001)$.
OUTCOME

No patient was physically harmed by being discharged from the accident department.

There was no significant difference in the waiting time for appointments at outpatient clinics and general practitioners' surgeries between the two groups, nor in duration of stay in psychiatric wards, or in number of attendances at clinics or surgeries or interviews with social workers. Of the 50 patients who did not receive the recommended treatment (table I), 14 (seven in each group) obtained some other form of treatment.

There were no suicides in either group. The number of patients who repeated the self-poisoning or self-injury within one year was identical in the two groups (table II). The repetition rate was $16 \%$ (18/115) for group D and $20 \%(20 / 98)$ for group $\mathrm{A}$ in those who came back to Addenbrooke's Hospital.

TABLE I-Treatment recommended. (Figures in parentheses are number who actually received treatment recommended)

\begin{tabular}{|c|c|c|}
\hline \multirow{2}{*}{ Treatment } & \multicolumn{2}{|c|}{ Groups } \\
\hline & $\mathrm{D}$ & A \\
\hline $\begin{array}{l}\text { Inpatient/day patient } \\
\text { Outpatient } \\
\text { Social work } \\
\text { General practitioner } \\
\text { None }\end{array}$ & $\begin{array}{l}16(16) \\
14(8) \\
17(9) \\
19(11) \\
28(27)\end{array}$ & $\begin{array}{l}13(12) \\
37(29) \\
25(13) \\
15(9) \\
3(3)\end{array}$ \\
\hline \multirow[t]{2}{*}{$\begin{array}{l}\text { Total } \\
\text { Self-discharge }\end{array}$} & $\begin{array}{l}94(71) \\
21\end{array}$ & $\underset{5}{93(66)}$ \\
\hline & 115 & 98 \\
\hline
\end{tabular}

TABLE II-Outcome after one year

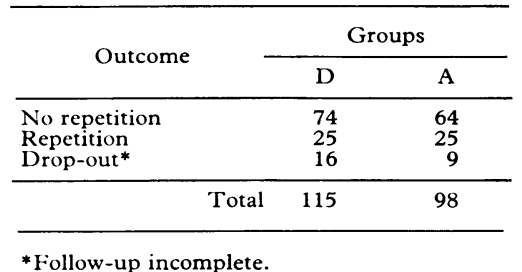

Patients who took their own discharge, though only $12 \%$ of the total, accounted for $20 \%$ of repetitions. They were the least co-operative patients, and if excluded the repetition rate for group $D$ is lower than that for group A, but not significantly so. Other less compliant patients, who did not receive the treatment recommended for them, were no more likely to repeat the episode than those who were treated. Statistically adjusted repetition rates at one year were $22.85 \%(S E \pm 4.02$ ) for group $D$ and $23.03 \%(S E \pm 4.30)$ for group $A$. There was also no apparent difference in the proportions of patients in the two groups surviving without repetition throughout the year. These rates include patients who, after repetition, were treated by general practitioners or other hospitals, or who failed to come to medical attention.

\section{Discussion}

Our results show that it is feasible for doctors who lack 2 specialist training in psychiatry to undertake evaluation of the less severely self-poisoned patient in an accident department. Ф The assumption ${ }^{12}$ that they would discharge patients whose? physical condition was satisfactory without taking psychological and social factors into account proved to be unfounded. They identified a small number of suicidal patients for informal admission to the psychiatric hospital. When they considered admission was necessary, or were uncertain about the suicidal risk or the need for psychiatric treatment, they admitted 8 patients to the medical wards-irrespective of their physical condition. Having selected for admission patients they considered 
most in need of help, the medical staff understandably offered further care to proportionately fewer of those sent home. Nevertheless, most patients they recommended for psychiatric treatment and aftercare, and discharged from the accident department, subsequently received it.

As expected, ${ }^{10}$ the least co-operative patients were the most likely to repeat their acts of self-poisoning. In contrast to Kennedy's retrospective study, ${ }^{11}$ the repetition rate of patients who were not admitted was no worse than in those admitted. It seems unlikely that patients who were discharged would have fared better if they had been admitted to a medical ward, because the amount of psychiatric treatment and aftercare given to the patients seen at this hospital matches that at the regional poisoning treatment centre in Edinburgh, ${ }^{12}$ which admits all patients, and the repetition rate is similar. ${ }^{13}$

Several factors may have contributed towards this outcome. In the accident department the nursing sisters helped to ensure that these patients received adequate attention. While the senior house officers lacked training in this work, the medical registrars (who saw two-thirds of the self-poisoned patients in the accident department) were experienced at making such assessments on the medical wards. A full-time social worker, who was mainly concerned with the admissions, was sometimes consulted. One psychiatrist (RG) had an outpatient clinic on site to facilitate referrals from wards and the accident department. At this hospital, staff attitudes were particularly favourable as a more selective approach had already been adopted towards the assessment of admissions, and junior doctors and nurses were suitably taught on the wards. ${ }^{5}{ }^{6}$

Since this study, each intake of casualty officers (and nursing staff) is given instruction in the psychological and social evaluation of self-poisoned patients ${ }^{14}$ and in the differential diagnosis of coma. The unit social worker devotes more time to the accident department, and we have tried to improve communication with the psychiatric and aftercare services, and with general practitioners. We think that such an approach will ensure that it is safe for junior medical staff not only to resuscitate selfpoisoned patients in the accident department, but also to decide the need for admission to hospital and for psychiatric or social work referral.

This project (No 56) was financed by the East Anglian Regional Health Authority. We thank the consultants at Addenbrooke's Hospital and at Fulbourn Hospital for permission to interview their patients; general practitioners for completing questionnaires; and Mrs M Gardner for collecting data.

\section{References}

${ }^{1}$ Ministry of Health. HM(61):94.

2 Joint Sub-Committee of the Standing Medical Advisory Committees. Hospital treatment of acute poisoning. London: HMSO, 1968.

3 Anonymous. Policies on self-poisoning. Br Med 7 1979;ii:1091-2.

${ }^{4}$ Anonymous. Overdose-will psychiatrist please see ? Lancet 1981;i:195-6.

5 Gardner R, Hanka R, O'Brien VC, Page AJF, Rees R. Psychological and social evaluation in cases of deliberate self-poisoning admitted to a general hospital. $\mathrm{Br}$ Med f 1977 ;ii:1567-70.

6 Gardner R, Hanka R, Evison B, Mountford PM, O'Brien VC, Roberts SJ. Consultation-liaison scheme for self-poisoned patients in a general hospital. Br Med F 1978;ii:1392-4.

7 Gardner R. Psychiatric assessment of self-poisoned patients in a general hospital. In: Murray R, Ghodse H, Harris C, Williams D, Williams P, eds. The misuse of psychotropic drugs. Brf Psychiatry 1981;41-4. (Special publication 1.)

${ }^{8}$ Black D, Pond D. Management of patients after self-poisoning. Br Med $\mathfrak{f}$ $1980 ; 280: 1141$

${ }^{9}$ Kreitman N. Services for parasuicide and the place of a poisonings unit. In: Farmer R, Hirsch S, eds. The suicide syndrome. London: Croom Helm, 1980:259-62.

${ }^{10}$ Greer S, Bagley C. Effect of psychiatric intervention in attempted suicide: a controlled study. $\mathrm{Br}$ Med $\mathcal{F} 1971 ; \mathrm{i}: 310-2$.

${ }^{11}$ Kennedy $P$. Efficacy of a regional poisoning treatment centre in preventing further suicidal behaviour. Br Med $\mathcal{F} 1972$ :iv :255-7.

${ }^{12}$ Holding TA, Buglass D, Duffy JC, Kreitman N. Parasuicide in Edinburgh -a seven year review 1968-74. Br f Psychiatry 1977:130:534-43.

${ }^{13}$ Buglass D, Horton J. The repetition of parasuicide: a comparison of three cohorts. Br f Psychiatry $1974 ; 125: 168-74$

${ }^{14}$ Gardner R. Psychiatric aspects of poisoning. In: Vale JA, Meredith TJ, eds. Poisoning: diagnosis and treatment. London: Update Books, 1981: 69-77.

(Accepted 26 November 1981)

\section{Retiring gracefully}

Giving lectures to the lay public is something you either enjoy or loathe. I confess the preparation is arduous. Once that is over I relish the human contact that results. My subject is "Health in retirement." The local technical college arrange a group of people for me to harangue every few months. The lecture is part of a course including finance, housing, hobbies, etc all connected with adjusting to retirement.

After the talk I invite questions. This is the really interesting bit. If the atmosphere is right one can gain valuable insights into people's feelings about facing up to the new programme that an end to work must bring and the need to "change gear" both mentally and physically. One gentleman asked me timidly whether he ought to give up a habit he had acquired over a lifetime. "What habit do you mean ?" I asked hesitantly. "Standing on my head," he replied. He proceeded there and then to give the astonished class a four-minute demonstration. I was reminded of Lewis Carroll:

"You are old, Father William," the young man said,

"And your hair has become very white;

And yet you incessantly stand on your head-

Do you think at your age, it is right ?"

Needless to say I assured him it was perfectly harmless. Perhaps, I thought, it might even nourish the aging cortex.

But my greatest surprise was after a talk in which I had warned that growing older might sometimes mean loss of a spouse. After the meeting an elderly man came up to me, all smiles. "You were wrong in my case," he said. "I have been a bachelor all my life. Now I have retired I have changed my mind. I am to be married in a few weeks' time." One might say that for him it was not, in Dr Johnson's words,
"The triumph of hope over experience," but the reverse $-s$ I HENDERSON SMITH, general practitioner, Huddersfield.

\section{Sir Thomas Browne and the Baroque}

The Royal College of Physicians did not seem the obvious place to seek an escapist hour on a bleak winter afternoon. I was agreeably surprised. Under the oil-painted eyes of august physicians one can be transported back into the World of Baroque. A glimpse of architectural grandeur, ornamental gardens, painting, and sculpture of this era and the preceding Mannerist period, are shown in the context of the life of Sir Thomas Browne, physician and meditative artist. 1982 is the tercentenary of his death and provides the stimulus for this exhibition. As an honorary Fellow of the College he cannot claim an eponymous disease but is well recognised for his significant contribution to contemporaneous literature.

Seventeenth- and eighteenth-century copies of his books, together with later edited editions, lie open, inviting inspection. These include his best known contemplative work Urn Burial, inspired by the excavation of a number of Roman burial urns in his native East Anglia. Its theme ?"Immortality and the manifold follies of man. . . ." His texts are interspersed with the work of contemporary artists. While the swan-neck of Parmigianino's Madonna, extravagant fountains at the Villa d'Este, and the flawless beauty of Bernini's sculpture may not seem wholly congruous, they do provide a fascinating background. Slow down 300 years, ponder on the mysteries of the quincunx, and visit this exhibition.-TESSA RICHARDS, London.

See also News item on Sir Thomas Browne festival at Norwich. 